\title{
A transient absorption study of allophycocyanin
}

\author{
Y J SHIU ${ }^{1}, \mathrm{~J} \mathrm{M} \mathrm{ZHANG}^{1}, \mathrm{M} \mathrm{HAYASHI}^{2}, \mathrm{~V} \mathrm{GULBINAS}^{3}, \mathrm{C} \mathrm{M} \mathrm{YANG}^{4}$, \\ and $\mathrm{S} \mathrm{HLIN}^{1} *$ \\ ${ }^{1}$ Institute of Atomic and Molecular Sciences, Academia Sinica, Taipei, \\ Taiwan, ROC \\ ${ }^{2}$ Center for Condensed Matter Sciences, National Taiwan University, Taipei, \\ Taiwan, ROC \\ ${ }^{3}$ Institute of Physics, A-Gostauto-12, LT-2600 Vilnius, Lithuania \\ ${ }^{4}$ Institute of Botany, Academia Sinica, Taipei, Taiwan, ROC \\ e-mail: yingjan@gate.sinica.edu.tw; shlin@po.iams.sinica.edu.tw
}

\begin{abstract}
Transient dynamics of allophycocyanin trimers and monomers are observed by using the pump-probe, transient absorption technique. The origin of spectral components of the transient absorption spectra is discussed in terms of both kinetics and spectroscopy. We find that the energy gap between the ground and excited states of the unexcited subunit of allophycocyanin monomer decreases via an interaction with another excited subunit. For allophycocyanin trimer, we find that the fast dynamics results from the fast internal conversion and the first excited state is the only one electronic state which can trap the final population.
\end{abstract}

Keywords. Allophycocyanin; transient absorption; induced absorption; stimulated emission; ground state bleaching; internal conversion.

\section{Introduction}

The phycobiliproteins, allophycocyanin (APC) and C-phycocyanin (C-PC), are presumably considered to play an important role in the energy transfer process of plants and algae. These proteins are open-chain tetrapyrrole-containing proteins located at the core of the phycobilisome in cyanobacteria. ${ }^{1}$ The X-ray crystal structure of the trimer aggregation state exhibits $\mathrm{C}_{3}$-symmetry. In particular, the structures of such proteins show a ring-like homotrimer of three monomers consisting of $\alpha$ and $\beta$-polypeptide monomers as has been already discussed in references. ${ }^{2-4}$

In this work, we shall obtain basic information on transient dynamics of APC trimers and monomers based on pump-probe, transient absorption measurements. An origin of spectral components of the transient absorption spectra will be discussed.

\section{Experimental setup}

The transient absorption (TA) experimental arrangement is shown in figure 1, and the ultrashort laser system is the same as used in our previous work. ${ }^{5}$ The pump energy is provided by the output of an OPA laser and attenuated to $30 \mathrm{~nJ}$ per pulse with a pulse duration time of 100 femtoseconds (fs) with a FWHM of $6 \sim 7 \mathrm{~nm}$ at a repetition rate of $20 \mathrm{kHz}$; while the probe beam is a continuum white light centered at $810 \mathrm{~nm}$ (460

*For correspondence 
$1200 \mathrm{~nm}, \sim 1 \mathrm{~nJ}$ ), which is generated by focusing a $810 \mathrm{~nm}$ laser light on a sapphire crystal. In front of the sample cell, the continuum white light is divided into two beams with equal energy; one is for a probe beam and another one is for a reference beam. The pump and probe beams are focused on the same spot of the sample in a quartz flow cell of $1 \mathrm{~mm}$ thickness and the reference beam is also focused on a different location in the flow cell. After the flow cell, the probe and reference beams are sent to a detector (Oriel MS257). Both probe and reference signals coming out from the monochromator and photomultiplier are directly sent to a personal computer for processing in the real time. After calibrating the absorbance intensity of probe beam by the reference beams, we obtain the difference absorbance $(\Delta A)$. In this case, $\Delta A$ is given as the absorbance difference between with and without pump beam. In the experiment, the APC, used herein, are isolated from cyanobacterium cultures of the Anabaean variabilis. The preparations and characterization methods for APC trimers and monomers are described in reference. ${ }^{6}$

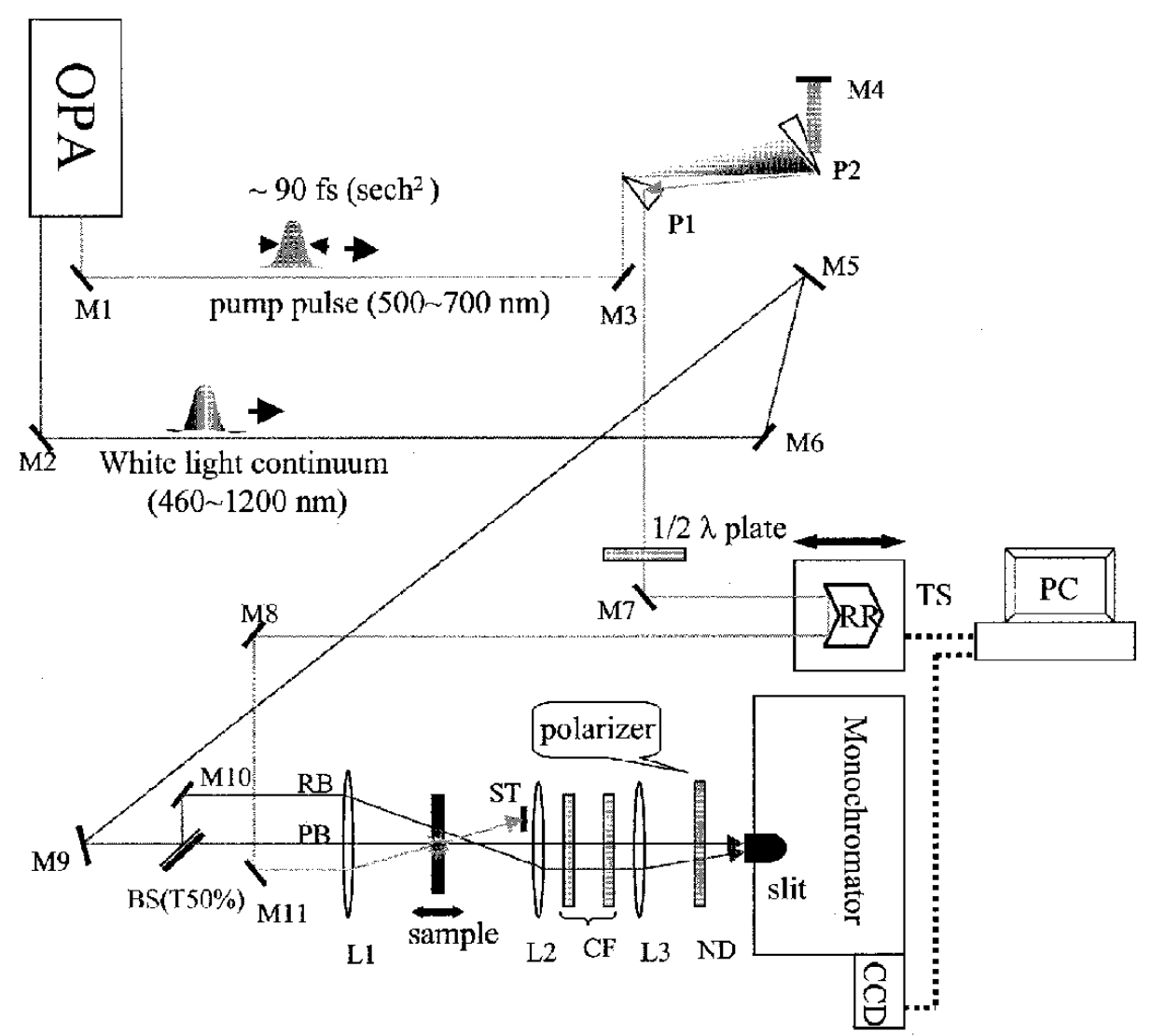

Figure 1. The experimental setup of transient absorption measurements. M: mirror, L: lens, P: prism, BS: beam splitter, TS: transition stage, RR: retro reflector, ST: stopper, ND: ND filter, CF: short cut off filter between 750 and $950 \mathrm{~nm}, \mathrm{~PB}$ : probe beam, RB: reference beam. 


\section{Results and discussions}

In the pump-probe system, generally, at least three types of optical processes exist (1) ground state bleaching (GB), (2) stimulated emission (SE), and (3) induced absorption (IA). The total signal of the difference absorbance is given by the summation of the three signals. In our transient absorption measurements, the IA signal is positive (+), while the GB and SE signals are negative (-). Figure 2 shows three-dimensional, transient absorption spectra of APC monomer (figure 2a) and APC trimer (figure 2b). Here APC trimers are excited at $580 \mathrm{~nm}$ with parallel polarization, while APC monomers are excited at $570 \mathrm{~nm}$ with magic angel $\left(54.7^{\circ}\right)$ polarization. Note that in figure $2 \mathrm{a}$ worse $\mathrm{S} / \mathrm{N}$ ratios at the pump wavelength $(\sim 570 \mathrm{~nm})$ can be seen, which is due to scattered light of the pump pulse. Figure 2 a shows a very slow decay component due to the population dynamics of the excited state during the observation time $\sim 150 \mathrm{ps}$. The strong IA bands at both the red and blue sides of the TA spectra appear immediately after the pump, and these bands almost persist without decreasing their intensities during the observed delay time. While
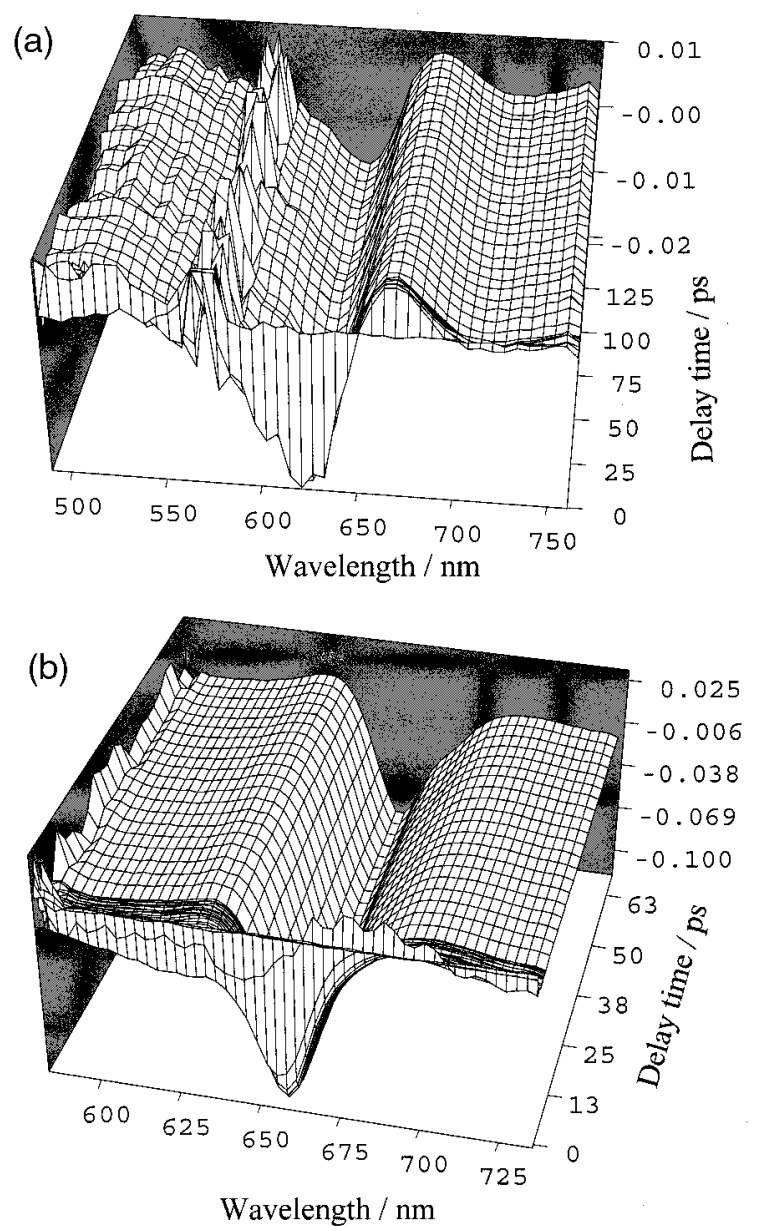

Figure 2. The observed three-dimensional, transient absorption spectra of APC monomer (a) and trimer (b). 
in figure $2 b$, the intensities of IA bands are found to decay with different time constants. Note that the intensity of IA bands depends on the pump power.

\subsection{TA spectra in frequency domain}

Figure 3 presents the observed transient absorption spectra of APC monomer (figure 3a) and trimer (figure $3 \mathrm{~b}$ ). The observed spectra are normalised at the maximum intensity in the negative intensity region and are shown as the function of probe wavelengths at several delay times. For comparison, the normalized absorption (dot line) and fluorescence (dash line) spectra are also shown in figure 3 . During $150 \mathrm{ps}$, figure 3 a exhibits a merged band of both the ground state bleaching and stimulated emission (GB/SE) located between the absorption and fluorescence spectral regions. From figure $3 \mathrm{a}$, one can see that the TA spectra are all the same during the delay time. On the other hand, the transient spectra of $\alpha$ subunit $^{7}$ excited at $620 \mathrm{~nm}$ are very similar to those of APC monomer (pumped at $570 \mathrm{~nm}$ ). The similarities indicate that the observed features of APC monomer simply result from individual $\alpha$ and $\beta$-subunits and these two chromophores weakly interact with each other.

(a)

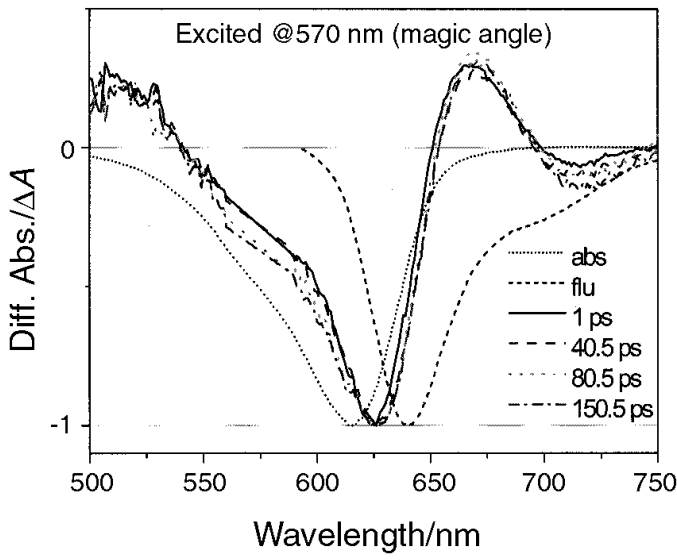

(b)

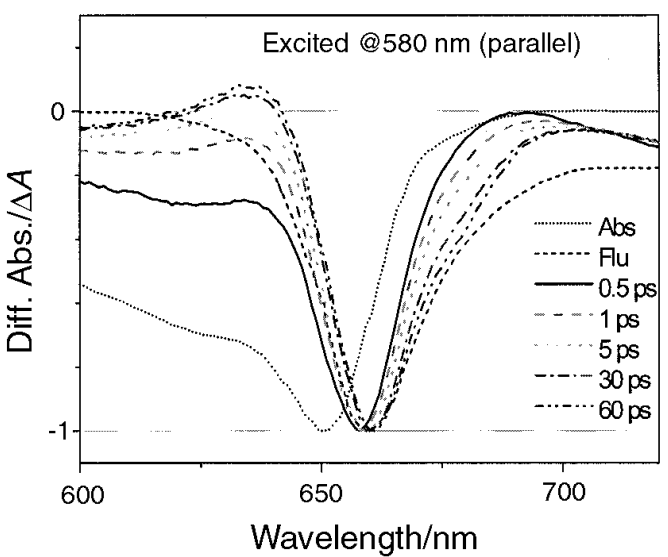

Figure 3. The normalized transient spectra at various delay times for APC monomer (a) and trimer (b). 
It is interesting to compare the monomer spectra with that of the APC trimer. From figure $3 \mathrm{~b}$, we notice that (1) the TA spectra within the $640 \sim 670 \mathrm{~nm}$ region provides the merged spectra of ground state bleaching and stimulated emission (GB/SE); (2) the $640 \sim 625 \mathrm{~nm}$ and 660 680 $\mathrm{nm}$ regions exhibit induced absorption (IA) bands; (3) the $580 \sim 620 \mathrm{~nm}$ region shows the GB spectra; and (4) the $700 \sim 720 \mathrm{~nm}$ region provides SE. It is found that the GB/SE merged band appears within 2 ps and it is located at the steadystate fluorescence region. This band exhibits a red shift with an increase of the delay time.

\subsection{The modeling of $A P C$}

To construct a microscopic model for the dynamics appearing in the observed time profiles of APC trimer and monomer, we attempt to extract IA spectra components by assuming that (1) GB and SE spectra components are directly associated with the steadystate absorption and fluorescence spectra and GB spectra components are independent of the delay time and (2) the blue and red region of TA spectra only contain GB and SE components.

3.2a APC monomer: We consider a microscopic model for APC monomer by assuming that the excitation takes place on $\alpha$ subunit or $\beta$-subunit is given by $\left.\left|\alpha_{g}\right\rangle\left|\beta_{g}\right\rangle \stackrel{\text { pump }}{\longrightarrow}\left|\alpha_{g}\right\rangle \beta_{e}\right\rangle$ or $\left|\alpha_{e}\right\rangle\left|\beta_{g}\right\rangle$. Note that two chromophores in the monomer are coupled weakly with each other. In our model, the electronic energies of $\left|\alpha_{z}\right\rangle\left|\beta_{c}\right\rangle$ and $\left|\alpha_{\varepsilon}\right\rangle\left|\beta_{g}\right\rangle$ are the same and only one subunit is excited. For example, excitation of $\boldsymbol{\alpha}$ subunit can lead to an interaction with the unexcited $\beta$-subunit, modifying the energy structure of the $\beta$-subunit. In this case, the energy gap between $\left|\alpha_{e}\right\rangle\left|\beta_{g}\right\rangle$ and $\left|\alpha_{e}\right\rangle\left|\beta_{e}\right\rangle$ (the second excited state in IA band) can be smaller. In other words, the net absorbance of TA spectra will show positive IA bands at the red side of the GB/SE band. This is due to the fact that the absorption spectra of chromophore in $\beta$-subunit is slightly shifted after the $\alpha$ subunit being excited.

Using two fitting rate constants, $1 / 25 \mathrm{ps}^{-1}$ and $1 / 300 \mathrm{ps}^{-1}$, as the initial parameters, we can describe the vibration relaxation rate constant $k_{r}$ of $|v\rangle$ and the electronic relaxation rate constant $k_{e}$ of $\left|\boldsymbol{\alpha}_{e}\right\rangle\left|\beta_{g}\right\rangle$, respectively. The population in each state is redefined as $\rho_{1}$ for the hot vibrational state $|v\rangle$ of $\left|\alpha_{\varepsilon}\right\rangle\left|\beta_{g}\right\rangle, \rho_{2}$ for the bottom of electronic state $\left|\alpha_{\varepsilon}\right\rangle\left|\beta_{g}\right\rangle$, and $\rho_{0}$ for the ground state $\left|\alpha_{g}\right\rangle\left|\beta_{g}\right\rangle$. Notice that $\mathrm{d} \rho_{1} / \mathrm{d} t=-k_{r} \rho_{1}$ and $\mathrm{d} \rho_{2} / \mathrm{d} t=k_{r} \rho_{1}-k_{e} \rho_{2}$. The absorbance $A$ consisting of the contributions from $\rho_{1}, \rho_{2}$ and $\rho_{0}$ can be written as $A=\left(\varepsilon_{g b}+\varepsilon_{g b}^{\prime}\right) \rho_{0}+\left(\varepsilon_{i a}^{\prime}-\varepsilon_{s e}^{\prime}\right) \rho_{1}+\left(\varepsilon_{i a}-\varepsilon_{s e}\right) \rho_{2}$, where $\rho=\rho_{1}+\rho_{2}+\rho_{0}$ and $\rho$ denotes the total concentration of APC monomer. Here, by ignoring the ground state dynamics after pumping, $\varepsilon_{g b}$ and $\varepsilon_{g b}^{\prime}$ can represent the absorption coefficients of GB for the states $\left|\boldsymbol{\alpha}_{e}\right\rangle\left|\boldsymbol{\beta}_{g}\right\rangle$ and $|v\rangle$, respectively. The terms $\varepsilon_{i a}$ and $\varepsilon_{i a}^{\prime}$ are the absorption coefficients of IA for the states $\left|\alpha_{\varepsilon}\right\rangle\left|\beta_{g}\right\rangle$ and $|v\rangle$, and $\varepsilon_{s e}$ and $\varepsilon_{s e}^{\prime}$ are the absorption coefficients of SE for the states $\left|\boldsymbol{\alpha}_{e}\right\rangle\left|\boldsymbol{\beta}_{z}\right\rangle$ and $|v\rangle$. Using the abovementioned equations $\Delta A=A-\bar{\varepsilon}_{g b} \rho=\left(\varepsilon_{i a}^{\prime}-\varepsilon_{s e}^{\prime}-\bar{\varepsilon}_{g b}\right) \rho_{1}+\left(\varepsilon_{i a}-\varepsilon_{s e}-\bar{\varepsilon}_{g b}\right) \rho_{2} \quad$ where $\bar{\varepsilon}_{g b}=\varepsilon_{g b}^{\prime}+\varepsilon_{g b}$. We can see that $\Delta A$ provides information on the time-development of the populations of $\rho_{1}$ and $\rho_{2}$ via the GB, SE, and IA processes. We assume that the coefficients of $\varepsilon_{g b}, \varepsilon_{g b}^{\prime}, \varepsilon_{i a}, \varepsilon_{i a}^{\prime}, \varepsilon_{s e}$ and $\varepsilon_{s e}^{\prime}$ are given by the Gaussian function, i.e. $\varepsilon_{i}=a_{i} \exp \left[-\left((\omega-\omega) / \Lambda_{i}\right)^{2}\right]$. Here $i$ is the index representing each coefficient component, $\Lambda_{i}$ is associated with the bandwidth, and $a_{i}$ is the corresponding maximum intensity of each band. All the parameters are listed in table 1. Using these parameters, we successfully 
simulate the three-dimensional transient absorption spectra as shown in figure 4 with a wavelength resolution of $5 \mathrm{~nm}$ and a time step of $1.5 \mathrm{ps}$. From figures $2 \mathrm{a}$ and 4 , good agreement can be seen between the simulation spectra and the observed spectra in both time and frequency domains. In the simulation, we use two Gaussian functions centered at $587.5 \mathrm{~nm}$ and $618.7 \mathrm{~nm}$ for the absorption spectra of GB band, the IA band at the red side of the GB/SE band is described by the ground state absorption spectrum with a red shift of $1200 \mathrm{~cm}^{-1}$, as shown in figure 5 . In the calculated spectrum in figure $5 \mathrm{~b}$, we use a ratio of $1: 1: 0.83$ for the GB, SE, and IA intensities, respectively. This IA spectrum is described by two Gaussian functions centered at $636.5 \mathrm{~nm}$ and $667.7 \mathrm{~nm}$. Here, the dynamics of the IA band at the blue side of GB/SE band is still unknown, we temporally describe this IA band with one Gaussian function centered at $515 \mathrm{~nm}$.

3.2b APC trimer: We find that our transient absorption spectra of APC trimer pumped at $580 \mathrm{~nm}$ agree with the reported in literature. ${ }^{8}$ An anisotropy profile reported in our previous work ${ }^{5}$ indicates that APC trimer should possess at least two electronically excited states in the visible region. The CD spectra, in fact, imply that the visible region of APC trimer involves at least three electronic states. However, the characteristic decay time of anisotropy profile is also found to be 200 500 fs upon excitations at $580 \mathrm{~nm}$ or $620 \mathrm{~nm}$ and probed at below $650 \mathrm{~nm}$ in this work. We could not obtain any anisotropy decay components when probed at shorter than $650 \mathrm{~nm}$. This phenomenon provides us with an idea that with an excitation at $580 \mathrm{~nm}$, hot vibrational states of the second electronic state are generated, from where fast internal conversion (IC) takes place within 200 500 fs.

For describing the three-dimensional, transient spectra of APC trimer, we need a model consisting of four states. In this model, subsequently to the excitation, the initial population is created in a hot vibrational state of the second excited electronic state $\left(\left|2_{v}\right\rangle\right)$ and via IC, the population relaxes from $\left|2_{v}\right\rangle$ to hot vibrational states of the first excited electronic state $\left(\left|1_{v^{\prime}}\right\rangle\right)$ and $\left(\left|1_{v^{\prime \prime}}\right\rangle\right)$. The vibrational states $\left|1_{v^{\prime}}\right\rangle$ and $\left|1_{v^{\prime \prime}}\right\rangle$ represent two vibrational modes with fast and slow relaxation rates respectively. Then the population relaxes

Table 1a. The list of center positions, bandwidths and relative intensities of APC monomer.

\begin{tabular}{|c|c|c|c|}
\hline & $\begin{array}{c}\text { SE } \\
\text { position/width/intensity }\end{array}$ & $\begin{array}{c}\text { IA } \\
\text { position/width/intensity }\end{array}$ & $\frac{\text { GB }}{\text { position/width/intensity }}$ \\
\hline $\begin{array}{l}|v\rangle \\
\text { vib. relax. }\end{array}$ & $\begin{array}{l}630 \cdot 5 \mathrm{~nm} / 18 \mathrm{~nm} / 0 \cdot 848 \\
680 \cdot 8 \mathrm{~nm} / 48 \cdot 2 \mathrm{~nm} / 0 \cdot 16\end{array}$ & $\begin{array}{l}515 \cdot 0 \mathrm{~nm} / 35 \mathrm{~nm} / 0 \cdot 4 \\
636.5 \mathrm{~nm} / 45 \cdot 7 \mathrm{~nm} / 0 \cdot 31 \\
667.7 \mathrm{~nm} / 23.4 \mathrm{~nm} / 0 \cdot 39\end{array}$ & $\begin{array}{l}587.5 \mathrm{~nm} / 45.7 \mathrm{~nm} / 0.529 \\
618.7 \mathrm{~nm} / 23.4 \mathrm{~nm} / 0.653\end{array}$ \\
\hline $\begin{array}{l}\left|\alpha_{e}\right\rangle\left|\beta_{g}\right\rangle \\
\text { elec. relax. }\end{array}$ & $\begin{array}{l}635 \cdot 5 \mathrm{~nm} / 21 \mathrm{~nm} / 0 \cdot 848 \\
680 \cdot 8 \mathrm{~nm} / 48 \cdot 2 \mathrm{~nm} / 0 \cdot 31\end{array}$ & $\begin{array}{l}515.0 \mathrm{~nm} / 35 \mathrm{~nm} / 0 \cdot 4 \\
636.5 \mathrm{~nm} / 45.7 \mathrm{~nm} / 0 \cdot 31 \\
667.7 \mathrm{~nm} / 23.4 \mathrm{~nm} / 0 \cdot 39\end{array}$ & \\
\hline
\end{tabular}

Table 1b. The list of time constants (ps).

\begin{tabular}{ll}
\hline $1 / k_{r}$ & $1 / k_{e}$ \\
25 & 300 \\
\hline
\end{tabular}




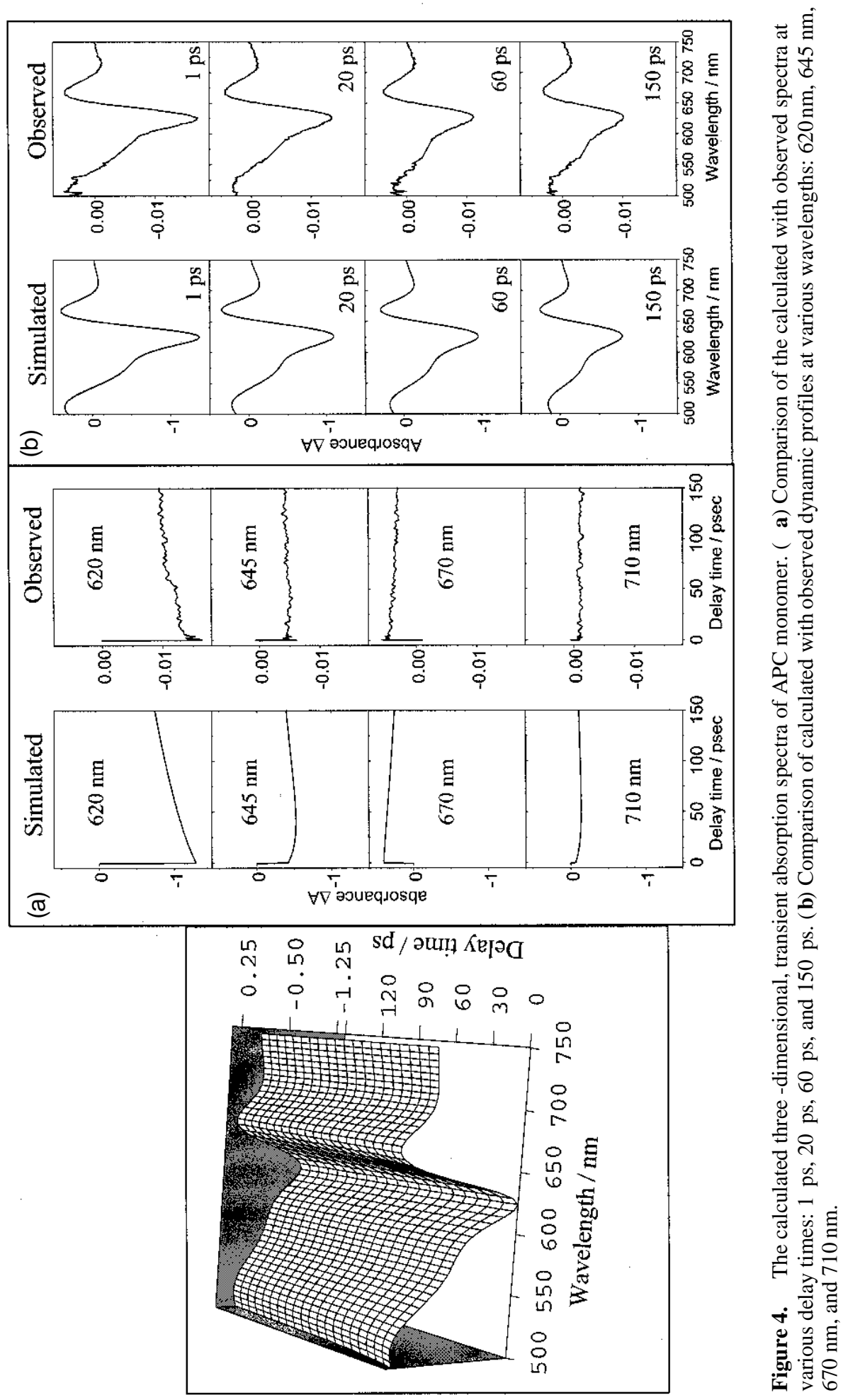



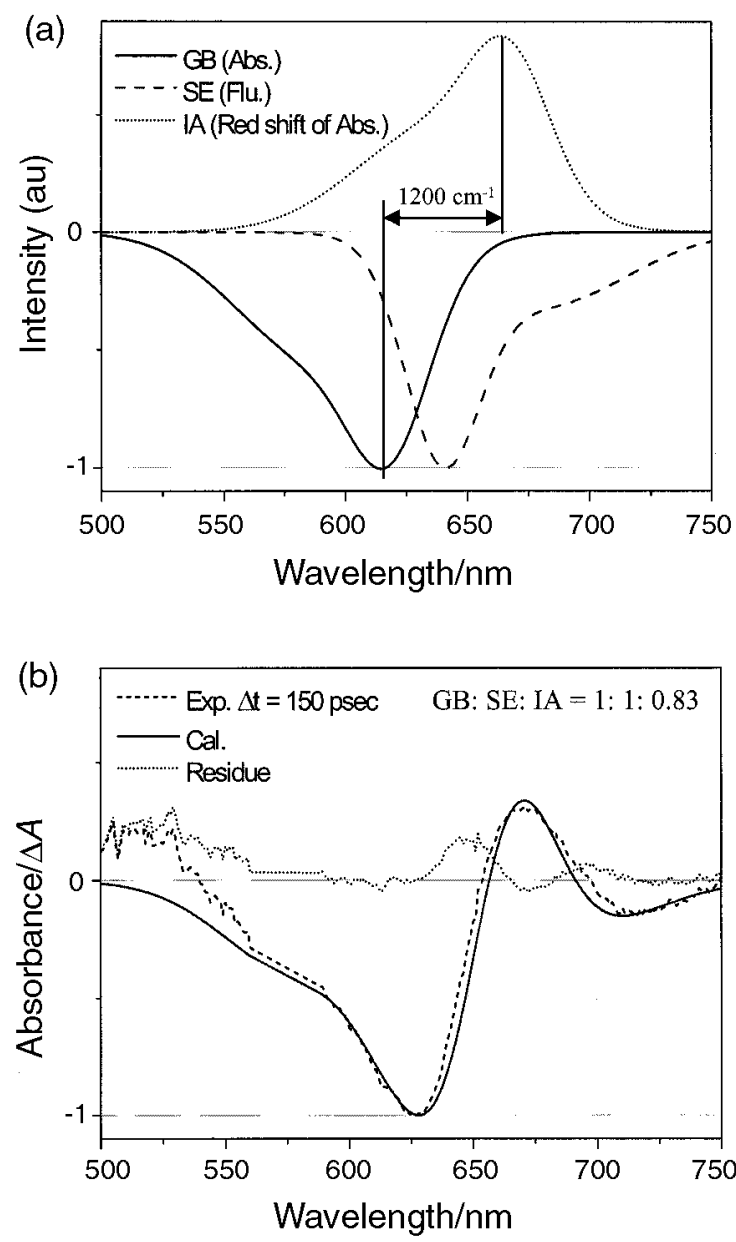

Figure 5. The model of IA spectra of APC monomer. (a) The absorption and fluorescence spectra of APC monomer are assumed to result from an excitation of one subunit in the monomer. (b) Comparison between the calculated and observed TA spectra.

from the hot vibrational states to the bottom of the first excited electronic state $(|1\rangle)$. Since the fast IC dominates in the initial relaxation process, nonradiative transition $\left(k_{n}\right)$ from a hot vibrational state to the bottom of the second excited electronic state $(|2\rangle)$ can be ignored. Three important rate constants $1 / 0 \cdot 2 \mathrm{ps}^{-1}, 1 / 2 \cdot 5 \mathrm{ps}^{-1}$, and $1 / 30 \mathrm{ps}^{-1}$ are found in our fitting. In our model, we assign the rate constants as the IC rate $k_{I C}$, vibrational relaxation rates $k_{r 1}$ (fast) and $k_{r 2}$ (slower) of $\left|1_{v^{\prime}}\right\rangle$ and $\left|1_{v^{\prime \prime}}\right\rangle$, respectively. The electronic lifetime $1 / k_{e}$ of the first excited state $|1\rangle$ is assumed to be longer than $10 \mathrm{~ns}$. For the trimer case, we have $\mathrm{d} \rho_{b b} / \mathrm{d} t=-k_{I C} \rho_{b b}, \mathrm{~d} \rho_{c c} / \mathrm{d} t=k_{I C} \rho_{b b}-k_{r 1} \rho_{c c}$, $\mathrm{d} \rho_{d d} / \mathrm{d} t=k_{I C} \rho_{b b}-k_{r 2} \rho_{d d}$, and $\mathrm{d} \rho_{e e} / \mathrm{d} t=k_{r 1} \rho_{c c}+k_{r 2} \rho_{d d}-k_{e} \rho_{e e}$. Thus, the difference absorbance in transient spectra $\Delta A$ can be written as 


$$
\begin{aligned}
\Delta A= & A-A_{0}=\left(\varepsilon_{i a 1}-\varepsilon_{s e 1}-\bar{\varepsilon}_{g b}\right) \rho_{b b}, \\
& +\left(\varepsilon_{i a 2}-\varepsilon_{s e 2}-\bar{\varepsilon}_{g b}\right) \rho_{c c}+\left(\varepsilon_{i a 3}-\varepsilon_{s e 3}-\bar{\varepsilon}_{g b}\right) \rho_{d d}, \\
& +\left(\varepsilon_{i a 4}-\varepsilon_{s e 4}-\bar{\varepsilon}_{g b}\right) \rho_{e e},
\end{aligned}
$$

where

$$
\begin{aligned}
& \bar{\varepsilon}_{g b}=\varepsilon_{g b 1}+\varepsilon_{g b 2}+\varepsilon_{g b 3}+\varepsilon_{g b 4}, \\
& A_{0}=\bar{\varepsilon}_{g b 1} \rho_{a a}(0),
\end{aligned}
$$

and

$$
\rho_{a a}(0)=\rho_{a a}+\rho_{b b}+\rho_{c c}+\rho_{d d}+\rho_{e e} .
$$

Here, $\boldsymbol{\rho}_{a a}, \boldsymbol{\rho}_{b b}, \boldsymbol{\rho}_{c c}, \boldsymbol{\rho}_{d d}$ and $\boldsymbol{\rho}_{e e}$ represent the populations of ground state, $\left|2_{\mathrm{v}}\right\rangle,\left|1_{v^{\prime}}\right\rangle$, $\left|1_{v^{\prime \prime}}\right\rangle$ and bottom of the first excited state. Figure 6 shows the calculated three-dimensional, TA spectra of APC trimer with a wavelength resolution of $3 \mathrm{~nm}$ and a time step of $0.3 \mathrm{ps}$, which can be compared with figure $2 \mathrm{~b}$. In the simulation, the GB band is described by three Gaussian functions centered at $652.3 \mathrm{~nm}, 634.5 \mathrm{~nm}$, and $602.7 \mathrm{~nm}$; the IA band is described by two Gaussian functions at $647 \mathrm{~nm}$ and $680 \mathrm{~nm}$. All the parameters are listed in table 2. It is interesting to note here that the $680 \mathrm{~nm}$ IA band results only from the second excited state $\left|2_{v}\right\rangle$ and the IA band at $647 \mathrm{~nm}$ appears at first excited state. The peak position of the SE band of the $\left|1_{v^{\prime \prime}}\right\rangle$ state exhibits a slightly red

Table 2a. The list of center positions, bandwidths and relative intensities of APC trimer.

\begin{tabular}{lccc}
\hline & $\begin{array}{c}\text { SE } \\
\text { position/width/intensity }\end{array}$ & $\begin{array}{c}\text { IA } \\
\text { position/width/intensity }\end{array}$ & $\begin{array}{c}\text { GB } \\
\text { position/width/intensity }\end{array}$ \\
\hline $\left.2_{v}\right\rangle$ & $730 \mathrm{~nm} / 36 \mathrm{~nm} / 0 \cdot 13$ & $680 \mathrm{~nm} / 30 \mathrm{~nm} / 0 \cdot 15$ & $\begin{array}{l}602 \cdot 7 \mathrm{~nm} / 44 \cdot 8 \mathrm{~nm} / 0 \cdot 12 \\
634 \cdot 5 \mathrm{~nm} / 30 \mathrm{~nm} / 0 \cdot 11\end{array}$ \\
& & & $652 \cdot 3 \mathrm{~nm} / 11 \cdot 6 \mathrm{~nm} / 0 \cdot 19$ \\
$\left|1_{v^{\prime}}\right\rangle$ & $658 \mathrm{~nm} / 10 \mathrm{~nm} / 1 \cdot 25$ & $647 \mathrm{~nm} / 20 \mathrm{~nm} / 0 \cdot 05$ & \\
& $675 \mathrm{~nm} / 10 \mathrm{~nm} / 0 \cdot 25$ & & \\
$\left|1_{v^{\prime \prime}}\right\rangle$ & $730 \mathrm{~nm} / 30 \mathrm{~nm} / 0 \cdot 187$ & & \\
& $659 \mathrm{~nm} / 12 \mathrm{~nm} / 1 \cdot 5$ & $647 \mathrm{~nm} / 20 \mathrm{~nm} / 0 \cdot 3$ & \\
$|1\rangle$ & $679 \mathrm{~nm} / 10 \mathrm{~nm} / 0 \cdot 2$ & & \\
& $730 \mathrm{~nm} / 30 \mathrm{~nm} / 0 \cdot 19$ & $647 \mathrm{~nm} / 20 \mathrm{~nm} / 0 \cdot 44$ & \\
& $660 \mathrm{~nm} / 12 \mathrm{~nm} / 1 \cdot 13$ & & \\
\hline
\end{tabular}

Table 2b. The list of time constants (ps).

\begin{tabular}{lccc}
\hline $1 / k_{I C}$ & $1 / k_{r l}$ & $1 / k_{r 2}$ & $1 / k_{e}$ \\
$0 \cdot 2$ & $2 \cdot 5$ & 30 & 10000 \\
\hline
\end{tabular}




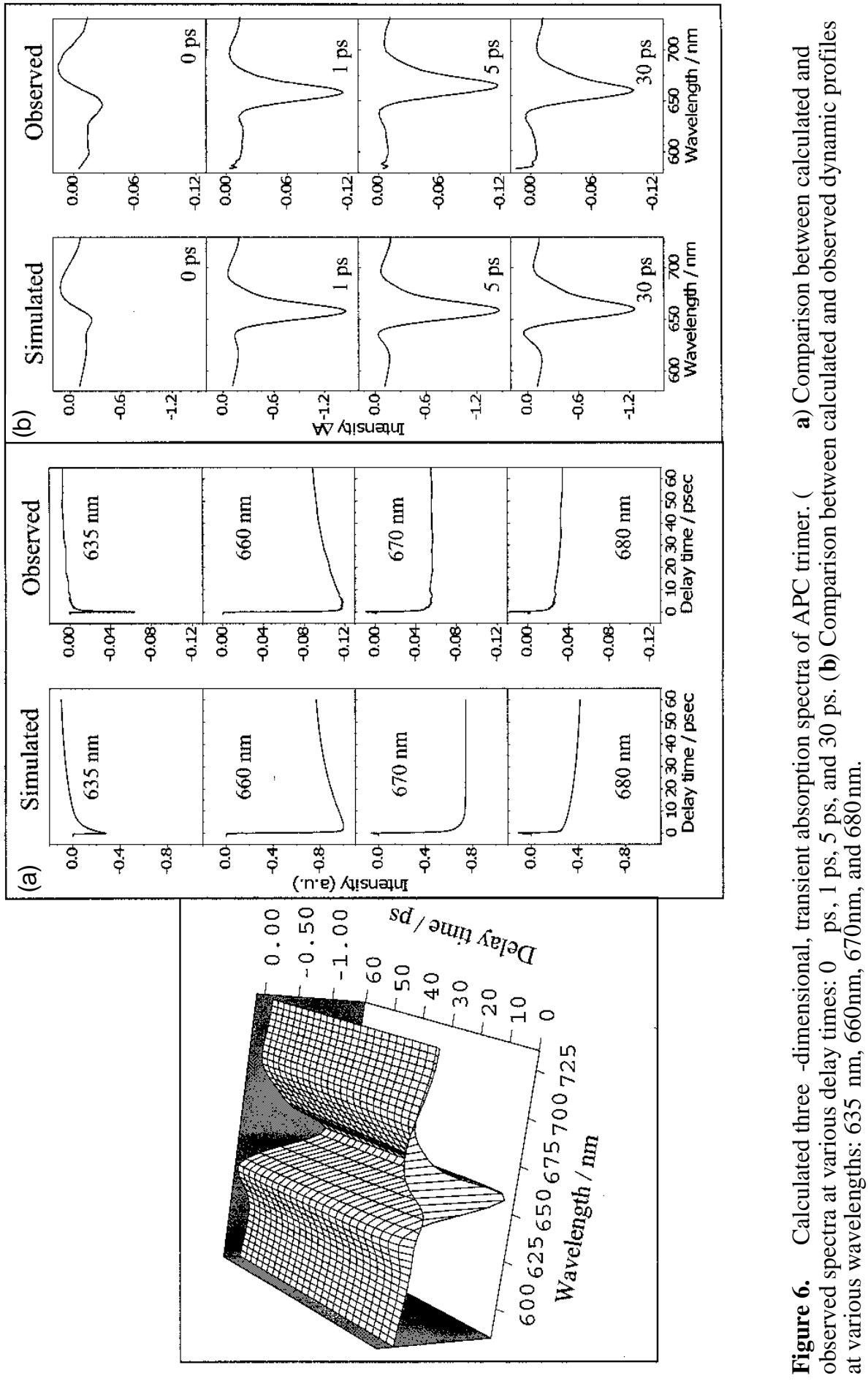


shift to $679 \mathrm{~nm}$. Figures 6 show a comparison between the simulation and observed spectra in both time and frequency domains and good agreement can be seen. We find that the fast dynamics of APC trimer results from IC process. Basically, six exciton states can exist once the electronic interactions among six chromophores are considered. However, through the fast non-radiative channel IC, no matter which state is excited the population will relax to the first excited state during 200 500 fs. The first excited state is the only one electronic state which can trap the final population.

\section{Conclusion}

We have shown our transient absorption spectra of APC. To understand the observed TA spectra, we have constructed a model for both the energy levels and the dynamics. Based on the proposed model, for the monomer case, we have obtained an IA band with a red shift of $1200 \mathrm{~cm}^{-1}$ from the ground state absorption band. This indicates that the energy gap between the ground and excited states of the unexcited subunit decreases by $1200 \mathrm{~cm}^{-1}$. For APC trimer, we have found that the excited population quickly arrives at the first excited state via IC, which allows us to observe the very fast dynamics within the early pumping time. In our model, we have attempted to use the minimum number of states to describe the all kinetics. We have found that for APC monomer, we need at least two excited states to explain the all processes while for APC trimer, at least four excited states are needed. The purpose of constructing model is not to reproduce the TA results accurately but to obtain the essence of dynamics. Our models can provide essential pictures for understanding the transient dynamics of APC trimer and monomers.

\section{Acknowledgments}

The authors wish to thank the Nan Pao Resins Chemical Co. Ltd. Bio. Dept. in Tainan, Taiwan, for providing us with cyanobacteria for preparation of APC sample. This work was financially supported by NSC of ROC, NNFC of PRC, and Academia Sinica of ROC.

\section{References}

1. Schirmer T, Bode W and Huber R 1987 J. Mol. Biol. 196677

2. McDemott G, Prince S M, Freer A A, Hawthornthwaite-Lawless A M, Papiz MZ, Cogdell R J and Isaacs N W 1995 Nature (London) 374517

3. Brejc K, Ficner R, Huber R and Steinbacher S 1995 J. Mol. Biol. 249424

4. Glazer A N 1985 Annu. Rev. Biophys. Biochem. 1447

5. Zhang J M, Shiu Y J, Hayashi M, Liang K K, Chang C H, Gulbinas V, Yang C M, Yang T-S, Wang H Z, Yit-Tsong Chen and Lin S H 2001 J. Phys. Chem. A105 8878

6. Zhang J M, Zhao J Q and Jiang L J 1998 Photochem. Phtobiol. 68777

7. Riter R E, Edington D Maurice and Beck W F 1996 J. Phys. Chem. 10014198

8. Edington Maurice D, Riter Ruth E and Beck Warren F 1996 J. Phys. Chem. 10014206 